\title{
Are multiple sclerosis therapies safe in SARS-Cov-2 times?
}

\author{
Francesco Ferrara ${ }^{1}$, Giovanni Granata ${ }^{2}$, Chiara Pelliccia $^{3}$, Raffaele La Porta ${ }^{4}$, and Antonio \\ Vitiello ${ }^{1}$ \\ ${ }^{1}$ Azienda Unità Sanitaria Locale Umbria 1 \\ ${ }^{2}$ Local Health Authority Salerno \\ ${ }^{3}$ Azienda Unità Sanitaria Locale Umbria 2 \\ ${ }^{4}$ ASUR Marche
}

May 11, 2020

\begin{abstract}
During the SARS-Cov-2 pandemic, it is essential to identify the risk factors that can cause a higher probability of infection and, therefore, worsen the patient's health. In fact, the known risk factors include already existing diseases and associated pharmacological treatments. A patient with multiple sclerosis takes immunomodulatory drugs and certainly has a high risk. Evidence and literature have shown that SARS-Cov-2 infection causes severe lung damage due to a poorly functioning immune system and overexpression of cytokines. Therefore the management of multiple sclerosis treatments in immunomodulating therapy must be carefully monitored. This article on the one hand analyzes and recalls the safety profile of all drugs for multiple sclerosis, on the other the recommendations adopted by different countries are highlighted, trying to understand if the suspension of MS treatment must actually materialize in order not to incur lethal covid pneumonia.
\end{abstract}

\section{Introduction}

SARS-CoV-2 spread rapidly throughout the world causing a global pandemic that put the entire population at risk. There are many people who have a risk of contracting the infection and above all those with preexisting comorbidities, the elderly or people with therapeutic treatments who can rapidly develop serious damage to the respiratory tract. SARS-CoV-2 infection can be divided into three phases: phase 1, asymptomatic period without hospitalization; phase 2, mild symptomatic period; phase 3 , severe symptomatic phase with high viral load and generalized hyperinflammatory state leading to lung damage. A sudden release of cytokines, "cytokine storm" (CS), characterizes phase 3 and is the most serious. For this reason, having an active immune system is very important in the first phase to fight the virus and in the last two phases to avoid respiratory damage. Treatments with immunomodulating drugs is a very high risk and for this reason patients with multiple sclerosis are more exposed to the possibility of infection. The correct management of the clinical pharmacological aspects associated with MS is of fundamental importance during the pandemic.

MS leads to severe neurological disability if not properly treated. It is an autoimmune disease with demyelinating lesions that cause a wide range of disabling symptoms. Multiple sclerosis is divided into various forms depending on the clinical pathology: benign, relapsing, transactional, predominantly progressive, secondarily progressive, progressive-renewing remission. The disease develops following the immune response against myelin. Multiple sclerosis (MS) exposes to a higher risk of infection (about $+40 \%$ ) than the general population, reducing the quality of life of patients with serious health risk. Frequent hospitalizations are important for respiratory and urinary tract infections. This is why it can be said that the MS patient is a fragile patient, and in this pandemic period, he must be monitored carefully. 
MS treatments are immunomodulating and include first-line drugs such as IFN-beta, glatiramer, dimethyl fumarate, teriflunomide and second-line drugs such as fingolimod, natalizumab, ocrelizumab, alemtuzumab, cladribine. Thanks to these treatments it is possible to control the disease for many years. Observational studies and post-marketing surveillance activities are useful to improve the safety profile of all drugs for MS in the absence of reliable data and studies. It is easy to imagine how drugs that inhibit the immune system can lead to adverse reactions with serious effects and high risk of pandemic infection (1-12).

\section{Safety profile of MS therapies}

There are many drugs approved for the treatment of MS that lead to good disease control and high patient adherence and compliance. Unfortunately, however, these drugs are not free from serious adverse reactions which in some cases are fatal. This is because there is little data on safety on newer drugs such as ocrelizumab. Alemtuzumab was in fact withdrawn from the market in Italy following serious adverse reactions with CMV reactivation. The safety profile of INF and glatiramer, on the other hand, has been evaluated in numerous studies, the most frequent adverse reactions recorded being the symptoms of the flu-like syndrome and the injection site reaction. This is why it is considered the safest drug. Fingolimod leads to serious adverse rations and the most common are infections, such as the urinary tract and lower respiratory tract infections. Such reactions have been shown to be due to the reduction of lymphocytes in the blood. The use of teriflunomide and dimethyl fumarate shows that these drugs lead to a slight reduction in the white blood cell count that could expose the patient to infectious risk. The adverse events of ocrelizumab concern allergic reactions for infusion, including itching, rash, urticaria and hypoglobulinemia and lymphocytopenia associated with secondary infections. Based on the present literature, post-marketing pharmacovigilance data and product $\mathrm{CPR}$, it is clear that there is an increased risk of infection associated with drugs for MS. The marked reduction in IgG and IgA leads to an increased risk of causing infection, so the drugs for MS that cause a high reduction in IgG lead to a marked risk of infection for the patient. Drugs such as Ocrelizumab Cladribina should definitely be avoided because of their marked lymphocytopenia (13-34).

\section{The recommendations of the major countries}

The various regulatory agencies in the different countries have taken very specific positions to regulate the intake of drugs for MS without risk to the patient during the period of Sars-CoV-2. The aim of the study is to summarize all the recommendations of each individual country and understand the orientation towards stopping treatments for MS. In general, no country recommends stopping treatment for MS and taking care to classify drugs based on risk (table 1 ).

Only IFNs and Glatiramer agree on all countries: INFs are safe and can also protect against the risk of covid infection. This is why they are safe treatments. The glatiramer is also considered safe, only in Italy is the position more prident. Everyone agrees to discontinue therapy or shift to IFN in the event of a covid infection. Of all the countries, only the USA gives the doctor a wide choice to understand if it is appropriate to continue the current therapy or not. The American FDA simply divided the drugs according to the risk but left the doctor the choice to understand the real risk based on the patient's clinical picture.

Describing the remaining drugs we have that first-line drugs (teriflunomide and dimethyl fumarate) should be continued and suspended in the event of covid injection and, in Germany and Canada, also in the case of severe lymphopenia. In Italy, the position is more prudent with regard to teriflunomide and the possible shift towards interferon therapies must be assessed.

Second-line therapy with fingolimod disagrees the various nations: in Germany and Canada, continuation of therapy is recommended by carefully evaluating the risk; in Italy the suspension or shift to IFNs is assessed, while in the UK the emphasis is also placed on the danger of rebaunding the MS in case of sudden suspension.

Depletive drugs (cladribine, ocrelizumab and alemtuzumab) should always be suspended or delayed for up to 6 months. All agencies agree. Only ocrelizumab in the UK can be used if considered essential. The only exception to this class of drugs is natalizumab. NTZ is considered as safe all over the world as IFNs therapies, only in Italy there is greater caution with immediate suspension in case of positive covid patients. 


\section{Conclusions}

SARS-Cov-2 has highlighted even more the serious safety problems of drugs for MS especially with regard to new immunomodulatory drugs with increased potential risks of infection. Is it safe to continue therapy during the pandemic? Based on the evidence in the literature, the position of the agencies in the different countries and given the complexity of the treatment of MS the best thing is that the decision is based on the individual condition of the patient. It is useless to hide that the therapy must be even more personalized and the patient carefully monitored. MS needs to be treated avoiding infection risks: the doctor's job is to maintain this balance by ensuring the greatest possible safety. Depletive therapies, which significantly affect the patient's immune system, safely lead to a decrease in lymphocytes and an associated risk of infection. However, failure to take therapies can lead the patient to have new disease attacks and for this there is no reason to stop without proven need. It is important to follow national and international guidelines and the positions of scientific societies and regulatory agencies because the therapy must be personalized as much as possible according to the clinical picture. Important for this is constant monitoring.

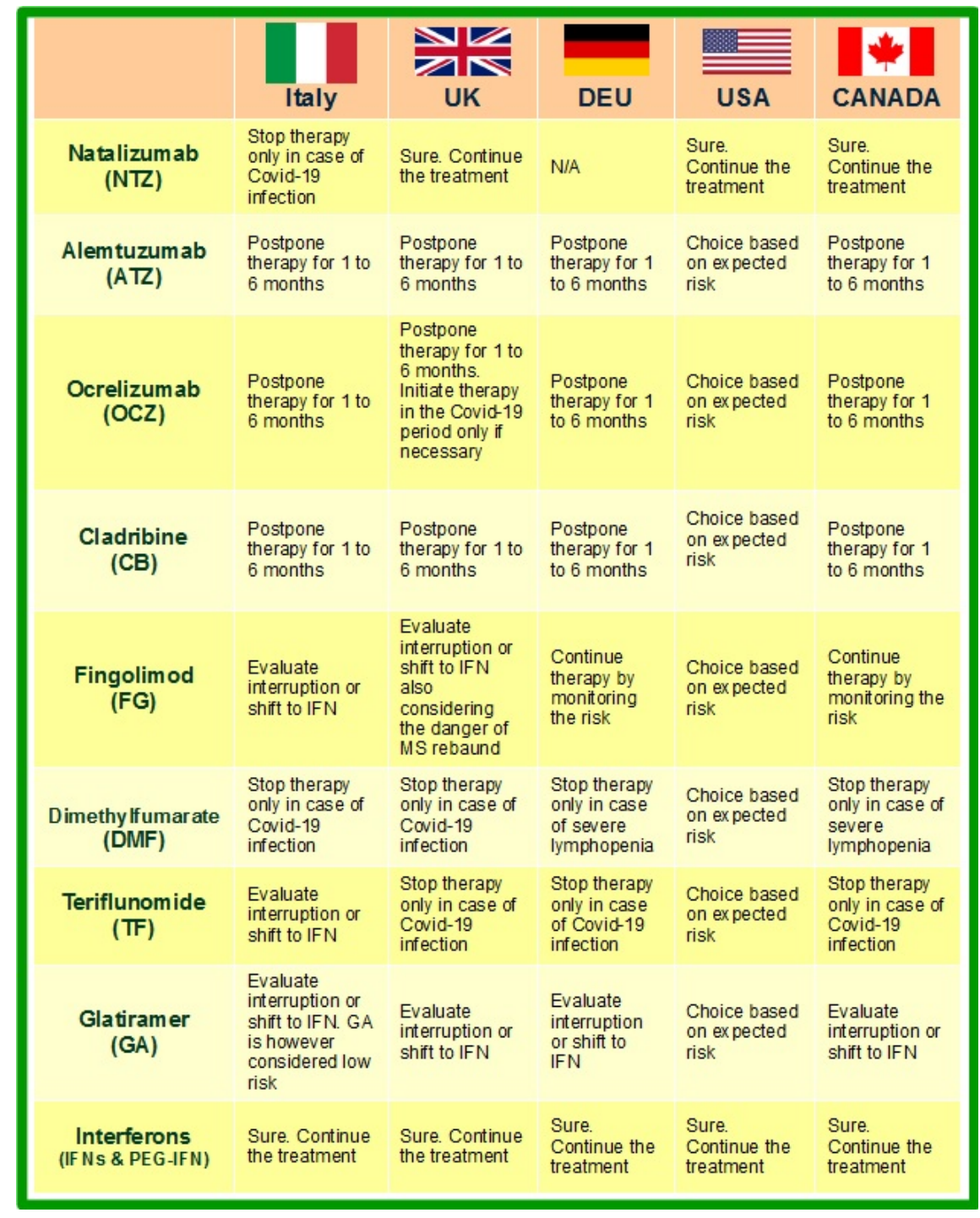

Table 1 Recommendations of the main world nations. 


\section{Main statements}

I, the undersigned, Francesco Ferrara and any other author, declare that:

- The manuscript was written entirely by the authors; · All authors made an equal contribution in the development of the paper; · We have no conflict of interest; · We have not received funding/source; · There are no sensitive data and no patients were recruited for this study; · The document does not conflict with ethical legislation.

Regards

The authors

\section{References}

1. 1.Guan WJ Ni ZY Hu Y et al.Clinical characteristics of coronavirus disease 2019 in China. $N$ Engl J Med. 2020; (published online Feb 28.)DOI:10.1056/NEJMoa2002032

2. .Zhang C Shi L Wang FS Liver injury in COVID-19: management and challenges. Lancet Gastroenterol Hepatol. 2020; (published online March)https://doi.org/10.1016/S2468-1253(20)30057-1

3. Huang C Wang Y Li X et al.Clinical features of patients infected with 2019 novel coronavirus in Wuhan, China.Lancet. 2020; 395: 497-506

4. Marrie RA et al. Dramatically changing rates and reasons for hospitalization in multiple sclerosis. Neurology. 2014; 83: 929- 937.

5. Montgomery S, Hillert J, Bahmanyar S. Hospital admission due to infections in multiple sclerosis patients. Eur J Neurol. 2013; 20: 1153- 1160.

6. Wijnands JM et al. Infection-related health care utilization among people with and without multiple sclerosis. Mult Scler. 2016; https://doi.org/10.1177/1352458516681198. Epub ahead of print

7. Smestad C, Sandvik L, Celius EG. Excess mortality and cause of death in a cohort of Norwegian multiple sclerosis patients. Mult Scler. 2009; 15: 1263- 1270.

8. Wijnands JMA et al. Health-care use before a first demyelinating event suggestive of a multiple sclerosis prodrome: a matched cohort study. Lancet Neurol. 2017; 16: 445- 451.

9. Bruck W et al. Therapeutic decisions in multiple sclerosis: moving beyond efficacy. JAMA Neurol. 2013; 70: 1315- 1324 .

10. Global, regional, and national life expectancy, all-cause mortality, and cause-specific mortality for 249 causes of death, 1980-2015: a systematic analysis for the Global Burden of Disease Study 2015. Lancet 2016; 388: 1459- 1544.

11. Williamson EM, Berger JR. Infection risk in patients on multiple sclerosis therapeutics. CNS Drugs. 2015; 29: 229- 244.

12. Andersen O, Lygner PE, Bergstrom T, Andersson M, Vahlne A. Viral infections trigger multiple sclerosis relapses: a prospective seroepidemiological study. J Neurol. 1993; 240: 417- 422.

13. Faissner S, Gold R Efficacy and Safety of the Newer Multiple Sclerosis Drugs Approved Since 2010. CNS Drugs. 2018 Mar;32(3):269-287. doi: 10.1007/s40263-018-0488.

14. Ellen Lu et al. Safety of disease-modifying drugs for multiplesclerosis in pregnancy: current challengesand future considerations for effectivepharmacovigilance. Expert Review of Neurotherapeutics.

15. Randomised double-blind placebo-controlled study of interferon beta-1a in relapsing/remitting multiple sclerosis. PRISMS (Prevention of Relapses and Disability by Interferon beta-1a Subcutaneously in Multiple Sclerosis) Study Group. Lancet Lond. Engl. 1998;352:1498-1504.

16. Clanet M, Radue EW, Kappos L, et al. A randomized, double-blind, dosecomparison study of weekly interferon beta-1a in relapsing MS.Neurology. 2002;59:1507-1517.

17. Sormani MP, Li DK, Bruzzi P, et al. Combined MRI lesions and relapses as a surrogate for disability in multiple sclerosis.Neurology. 2011;77:1684-1690.

18. Comi G, Filippi M, Barkhof F, et al. Effect of early interferon treatment on conversion to definite multiple sclerosis: a randomised study. Lancet Lond. Engl. 2001;357:1576-1582.

19. Davis MD, Ashtamker N, Steinerman JR, et al. Time course of glatiramer acetate efficacy in patients 
with RRMS in the GALA study. Neurol. Neuroimmunol. Neuroinflammation [Internet]. 2017 [cited 2019 Jul 25];

20. Johnson KP, Brooks BR, Cohen JA, et al. Copolymer 1 reduces relapse rate and improves disability in relapsing-remitting multiple sclerosis: results of a phase III multicenter, double-blind placebo-controlled trial. The Copolymer 1 Multiple Sclerosis Study Group. Neurology. 1995;45:1268-1276.

21. Comi G, Martinelli V, Rodegher M, et al. Effect of glatiramer acetate on conversion to clinically definite multiple sclerosis in patients with clinically isolated syndrome (PreCISe study): a randomised, double-blind, placebo-controlled trial. Lancet Lond. Engl. 2009;374:1503-1511.

22. D'Amico E, Leone C, Caserta C, et al. Oral drugs in multiple sclerosis therapy: an overview and a critical appraisal. Expert Rev. Neurother. 2015;15:803-824.

23. Oh J, O'Connor PW. Teriflunomide in the treatment of multiple sclerosis: current evidence and future prospects. Ther. Adv. Neurol. Disord. 2014;7:239-252.

24. O'Connor P, Wolinsky JS, Confavreux C, et al. Randomized trial of oral teriflunomide for relapsing multiple sclerosis. N. Engl. J. Med. 2011;365:1293- 1303.

25. Confavreux C, O'Connor P, Comi G, et al. Oral teriflunomide for patients with relapsing multiple sclerosis (TOWER): a randomised, double-blind, placebocontrolled, phase 3 trial. Lancet Neurol. 2014;13:247-256.

26. Miller AE, Wolinsky JS, Kappos L, et al. Oral teriflunomide for patients with a first clinical episode suggestive of multiple sclerosis (TOPIC): a randomised, doubleblind, placebo-controlled, phase 3 trial. Lancet Neurol. 2014;13:977-986.

27. O'Connor P, Comi G, Freedman MS, et al. Long-term safety and efficacy of teriflunomide: Nine-year follow-up of the randomized TEMSO study. Neurology. 2016;86:920-930. [

28. Linker RA, Haghikia A. Dimethyl fumarate in multiple sclerosis: latest developments, evidence and place in therapy. Ther. Adv. Chronic Dis. 2016;7:198- 207.

29. Diebold M, Altersberger V, Decard BF, et al. A case of progressive multifocal leukoencephalopathy under dimethyl fumarate treatment without severe lymphopenia or immunosenescence. Mult. Scler. Houndmills Basingstoke Engl. 2019;1352458519852100.

30. Safety and efficacy of fingolimod in patients with relapsing-remitting multiple sclerosis (FREEDOMS II): a double-blind, randomised, placebo-controlled, phase 3 trial. Lancet Neurol. 2014;13:545-556.

31. Cohen JA, Khatri B, Barkhof F, et al. Long-term (up to 4.5 years) treatment with fingolimod in multiple sclerosis: results from the extension of the randomised TRANSFORMS study. J. Neurol. Neurosurg. Psychiatry. 2016;87:468-475.

32. Cohen JA, Chun J. Mechanisms of fingolimod's efficacy and adverse effects in multiple sclerosis. Ann. Neurol. 2011;69:759-777.

33. Giovannoni G, Comi G, Cook S, et al. A placebo-controlled trial of oral cladribine for relapsing multiple sclerosis. N. Engl. J. Med. 2010;362:416-426.

34. Cook S, Vermersch P, Comi G, et al. Safety and tolerability of cladribine tablets in multiple sclerosis: the CLARITY (CLAdRIbine Tablets treating multiple sclerosis orallY) study. Mult. Scler. Houndmills Basingstoke Engl. 2011;17:578-593 